Louisiana State University

LSU Digital Commons

$4-1-2008$

\title{
Photothermal deflection determination of iron(II) with ferrozine with sorption preconcentration on silufol plates
}

\author{
A. D. Khrycheva \\ Lomonosov Moscow State University \\ D. A. Nedosekin \\ Lomonosov Moscow State University \\ M. A. Proskurnin \\ Lomonosov Moscow State University \\ M. Y. Kononets \\ Lomonosov Moscow State University
}

S. V. Pakhomova

P.P.Shirshov Institute of Oceanology, Russian Academy of Sciences

See next page for additional authors

Follow this and additional works at: https://digitalcommons.Isu.edu/biosci_pubs

\section{Recommended Citation}

Khrycheva, A., Nedosekin, D., Proskurnin, M., Kononets, M., Pakhomova, S., \& Faubel, W. (2008).

Photothermal deflection determination of iron(II) with ferrozine with sorption preconcentration on silufol plates. Applied Spectroscopy, 62 (4), 450-457. https://doi.org/10.1366/000370208784046821

This Article is brought to you for free and open access by the Department of Biological Sciences at LSU Digital Commons. It has been accepted for inclusion in Faculty Publications by an authorized administrator of LSU Digital Commons. For more information, please contact ir@lsu.edu. 
Authors

A. D. Khrycheva, D. A. Nedosekin, M. A. Proskurnin, M. Y. Kononets, S. V. Pakhomova, and W. Faubel 


\title{
Photothermal Deflection Determination of Iron(II) with Ferrozine with Sorption Preconcentration on Silufol Plates
}

\author{
A. D. KHRYCHEVA, D. A. NEDOSEKIN, M. A. PROSKURNIN,* M. Y. KONONETS, \\ S. V. PAKHOMOVA, and W. FAUBEL \\ Chemistry Department, M.V. Lomonosov Moscow State University, Leninskie Gory d. 1 Bldg. 3, 119991 GSP-1 Moscow, Russia (A.D.K., D.A.N., \\ M.A.P., M.Y.K.); P.P. Shirshov Institute of Oceanology, Russian Academy of Sciences, Nakhimovskii prosp. 36, 117997 Moscow, Russia (S.V.P.); \\ and Research Center Karlsruhe, Institute of Technical Chemistry, Water Technology and Geotechnology Division, P.O. Box 3640, D-76021 \\ Karlsruhe, Germany (W.F.)
}

\begin{abstract}
Photothermal deflection spectroscopy was applied to the selective detection of iron(II) chelate with ferrozine by its sorption preconcentration on Silufol plates. The linearity range was $1 \times 10^{-11}-6 \times 10^{-8} \mathrm{~mol}$ $\mathrm{cm}^{-2}$ of chelate at the plate surface, which corresponded to $1 \times 10^{-9}-4 \times$ $10^{-6} \mathrm{M}$ of chelate in solution. The limits of detection and quantification are $8 \times 10^{-12}$ and $2.5 \times 10^{-11} \mathrm{~mol} \mathrm{~cm}{ }^{-2}$ at the plate from $15 \mu \mathrm{L}$ of test solution (0.5 $\mathrm{nM}$ and $1.5 \mathrm{nM}$ in solution, respectively), and the absolute detection limit is $8 \mathrm{fmol}$ in the whole spot applied to a plate. Characteristics and features of photothermal deflection detection are discussed.
\end{abstract}

Index Headings: Laser; Photothermal effects; Photothermal-deflection spectroscopy; Trace determination; Iron(II).

\section{INTRODUCTION}

Photothermal (beam) deflection (mirage effect) spectroscopy (PDS) is a very powerful tool for studying absorption profiles of various surfaces ${ }^{1}$ and provides measurements of thermal and physical properties and the thickness of a solid sample in a simple, inexpensive, and nondestructive way. PDS belongs to the group of photothermal methods introduced by Fournier and Boccara. ${ }^{2}$ The principle of this technique is the measurement of a laser probe beam deflection, which corresponds to the periodic thermal diffusion from a periodically heated sample into the gas adjacent to the sample surface. This modulated sample heating induced by means of a second laser, whose modulated excitation beam impinges on the sample surface, leads to a temperature-dependent density oscillation in the gas phase and thus to a periodic variation of its index of refraction. These changes in the index of refraction of the gas, usually air, can be indicated by the deflection of the probe beam skimming the sample surface and measured by a position-sensitive optical detector.

Moreover, PDS possesses high instrumental sensitivity and spatial resolution due to the use of focused laser radiation for sample excitation. Currently, PDS is one of the most suitable and widely used photothermal methods for imaging of surfaces

Received 2 November 2007; accepted 23 January 2008.

* Author to whom correspondence should be sent. E-mail: michael@ analyt.chem.msu.ru. having either extremely high or low absorption levels. Nowadays, it is usually applied to microscopic and defectoscopic studies of semiconductors, ${ }^{1}$ metals, ${ }^{3,4}$ and various optical materials. ${ }^{5,6}$ However, its application in analytical chemistry as a highly sensitive method of molecular absorption spectroscopy is not typical.

The existing analytical applications of the method ${ }^{7-9}$ include the use of PDS for immunoassay with the isolation of a specially colored antigen-antibody complex at the surface of a solid substrate and following photothermal detection of surface absorption. Several applications ${ }^{10-12}$ consider a specialized use of PDS in thin-layer chromatography with the detection of the whole lateral profile along the chromatogram development direction. A more recent application of PDS for thin-layer chromatographic analysis ${ }^{13}$ is limited to the measurements under non-selective excitation conditions. However, despite these valuable examples, PDS was not considered as a standalone technique of surface absorption detection, and the study ${ }^{13}$ was aimed only at the estimation of the depth profile of the spot concentration based on the determination of its thermal properties by several photothermal methods, with PDS among them.

Currently, there are few applications of PDS for the detection of colored substances adsorbed at the surface of an appropriate substrate. However, this method is very promising because it can use all the analytical experience of molecular spectroscopic analysis along with highly sensitive photothermal detection. Thus, simple and convenient analytical procedures may be developed using existing spectrophotometric procedures for solid-state and solution analysis. This strategy proved very useful for another widespread photothermal method, thermal lensing. ${ }^{14}$

In selecting the main target application, we considered the fact that trace determination of metals, particularly iron, in seawater is one of the topical tasks of marine chemistry. Iron is a very active participant in various biological and redox processes in seawater. ${ }^{15}$ Its concentration in seawater is very low and often lies at a level down to picomolar concentrations. ${ }^{15-19}$ Very few methods are suitable for the investigation of such a low level of iron. ${ }^{17,18}$ Methods of molecular absorption spectroscopy are among those suitable and have 


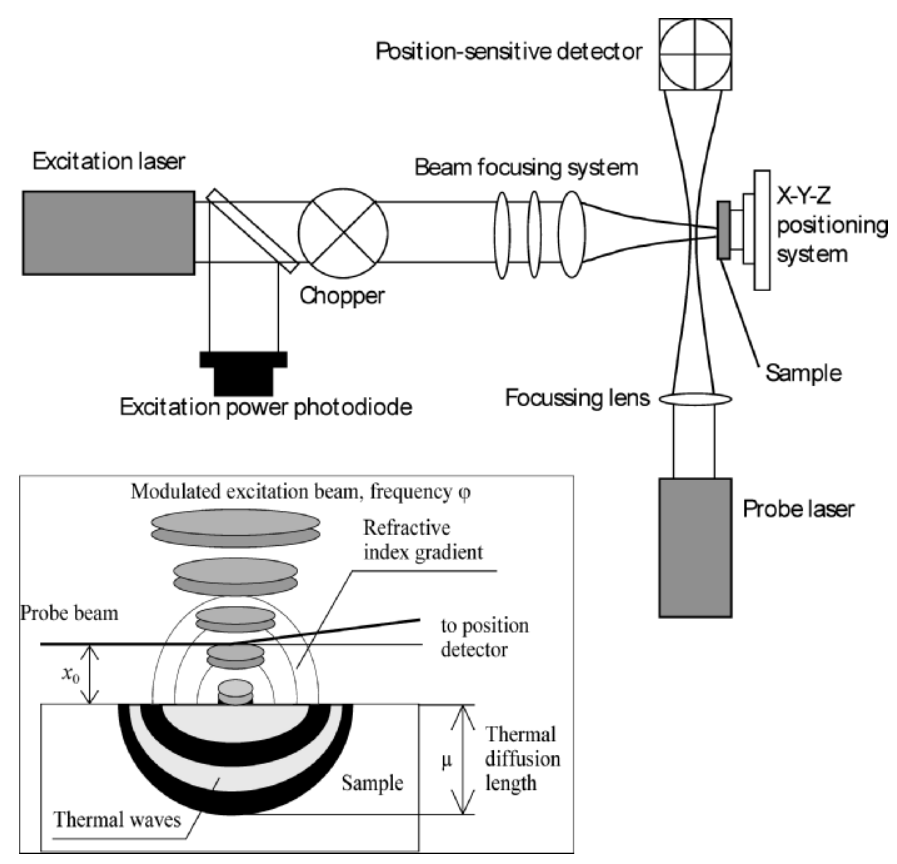

FIG. 1. The schematics of the photothermal deflection setup used and (inset) the principles and main parameters. See text for details.

an important feature of iron speciation analysis. ${ }^{17,18}$ However, as a rule, the sensitivity of spectrophotometric procedures is insufficient and needs to be improved. Photothermal deflection spectroscopy can be readily used for this task.

Hence, the aim of this work is to develop an easy and sensitive procedure for iron determination at a nanomolar level using PDS on the basis of existing photometric procedures. To facilitate photothermal-deflection spectroscopy, the metal was concentrated on a Silufol substrate followed by the on-substrate reaction with a sensitive and selective photometric reagent.

\section{MATERIALS AND METHODS}

Apparatus. The main principle of this technique is based on the measurement of a laser probe beam deflection, which corresponds to the periodic thermal diffusion from a periodically heated sample into the gas adjacent to the sample surface. ${ }^{2}$ A block scheme of the photothermal beam deflection (PBD) experimental arrangement, which has been described earlier in detail, is shown in Fig. 1. Sample heating was performed using a Nd: YAG laser (ADLAS DPY 321, Lubeck, Germany), wavelength $532 \mathrm{~nm}$ (with a frequency doubler for the $1064 \mathrm{~nm}$ basic laser line), and power $P_{\mathrm{e}}=50 \mathrm{~mW}$. The excitation beam has been focused on to the sample by means of optical components, and the radius of the excitation laser beam at the sample $\omega_{0 \mathrm{e}}$ was $100 \mu \mathrm{m}$. The excitation laser beam was modulated with a mechanical chopper with a frequency of 10$1000 \mathrm{~Hz}$, which corresponds to heating of a layer with a thickness $\mu$ (Fig. 1, inset) in the range from $1 \mu \mathrm{m}$ to $1 \mathrm{~nm}$. From the layer thickness and the reproducibility of measurements, ${ }^{3}$ the chopping frequency for analytical measurements was set to $\varphi=20 \mathrm{~Hz}$, which provided the signal from the sorbent layer only.

Probing was implemented with a continuous wave (cw) HeNe laser (Uniphase, CA), wavelength $632.8 \mathrm{~nm}$, power 5 $\mathrm{mW}$, and radius $60 \mu \mathrm{m} .^{3,20-22}$ The probe beam offset $x_{0}$ could be varied from $100 \mu \mathrm{m}$ up to $250 \mu \mathrm{m}$, and according to previous investigations $\mathrm{s}^{3,20-22}$ the surface-to-probe distance (Fig. 1, inset) was set to $x_{0}=100 \mu \mathrm{m}$. The signal was measured by a four-quadrant position-sensitive detector using a lock-in amplifier. The beam intensity profiles and waist sizes for both beams were estimated using an $\mathrm{M}^{2}-200$ Advanced PC Beam Propagation Analyzer (Spiricon ${ }^{\circledR}$ Inc., Logan, UT), with a precision of $5 \%$. The instrument was equipped with translation stages providing the imaging of $2 \times 2 \mathrm{~cm}$ samples with a step resolution of $1-20 \mu \mathrm{m}$.

The theoretically expected PDS signal amplitude $\theta$ was calculated from ${ }^{23,24}$

$$
\theta=-\frac{\mathrm{d} n}{\mathrm{~d} T} \frac{\alpha l P_{\mathrm{e}}}{\pi^{2} \varphi \rho C_{P} \omega_{\mathrm{e} 0}^{2}}\left(-\frac{2 x_{0}}{\omega_{\mathrm{e} 0}^{2}}\right) \exp \left(-\frac{x_{0}^{2}}{\omega_{\mathrm{e} 0}^{2}}\right)
$$

where $\mathrm{d} n / \mathrm{d} T$ is the refractive index gradient; $\alpha$ is the linear absorption coefficient of the light-absorbing substance; the detection is made over a line $l$ along the path defined by the illuminated region of the surface, where $l$ is the interaction pathway between the probe beam and the temperature gradient; $l$ is the thickness of the layer giving rise to the photothermal deflection signal; $\varphi$ is the chopping frequency; $\rho$ is density; $C_{\mathrm{p}}$ is heat capacity; and the parameters $P_{\mathrm{e}}, x_{0}$, and $\omega_{0 \mathrm{e}}$ are described above. The values of the thermo-optical parameters were taken from Ref. 25.

Reagents and Solvents. The following reagents were used throughout this work: ferrozine, monosodium salt $97 \%$ (Aldrich, Cat. no. 16,060-1), a $1 \times 10^{-2} \mathrm{M}$ stock solution, hydrochloric and glacial acetic acid (Reakhim, Russia, chemically pure grade), and sodium acetate trihydrate (Reakhim, Russia, high-purity grade $2-5$ ) were used to prepare $1: 1$ acetic buffer solutions by adding $6.3 \mathrm{~mL}$ of the acid to $13.6 \mathrm{~g}$ of the salt and adjusting the total volume to $100 \mathrm{~mL}, \mathrm{pH} 4.7$. Ascorbic acid (pharmaceutical grade), $25 \mathrm{~g} \mathrm{~L}^{-1}$ solutions; ammonium-iron(II) sulfate hexahydrate (Reakhim, Russia, analytical reagent grade), and $0.1000 \mathrm{mg} \mathrm{cm}^{-3}$ iron(III) solutions (GSO 7784-2000 certified reference material, Russian State Standard) were used throughout. All the solutions were prepared using Milli-Q water (specific resistance not lower than $18 \mathrm{M} \Omega / \mathrm{cm} \mathrm{Fe}, 2$ ppt $\left(3.6 \times 10^{-11} \mathrm{M}\right)$; dissolved $\mathrm{SiO}_{2}, 3 \mathrm{ppb}$; total ion amount, $<0.2 \mathrm{ppb}$; TOC, $<10 \mathrm{ppb}$ ) prior to the experiments. Stock solutions of ascorbic acid and sodium acetate were prepared every week; all the working solutions were prepared daily before the experiments. Solutions were filtered through a $0.45 \mu \mathrm{m}$ membrane filter. Hydrochloric acid was purified by isothermal distillation; the concentration of the resulting solution was controlled by titrimetry. Reagent-grade chloroform required for reagent purification was purified by distillation with calcium chloride (b.p. $61{ }^{\circ} \mathrm{C}$ ). After the distillation process, a small amount of ethanol was added (1 $\mathrm{mL}$ per $100 \mathrm{~mL}$ of chloroform) to avoid phosgene formation. ${ }^{26}$ The concentration of iron in all the used reagents was controlled with a Perkin-Elmer 603 atomic-absorption spectrometer (a 100-fold preconcentration according to the procedure found in Ref. 27). Ferrozine was purified by the following procedure. ${ }^{28}$

Ferrozine Purification Procedure. A $0.5 \mathrm{~mL}$ portion of the ascorbic acid solution $\left(25 \mathrm{~g} \mathrm{~L}^{-1}\right)$ and $0.5 \mathrm{~mL}$ of the acetic buffer solution were added to $5 \mathrm{~mL}$ of a ferrozine solution $(5 \times$ $10^{-3} \mathrm{M}$ ) in a separation funnel. The solution was kept for 15 min. Next $1 \mathrm{~mL}$ of $30 \%$ sodium perchlorate and $10 \mathrm{~mL}$ of chloroform were added to the funnel and it was slightly shaken for $1 \mathrm{~min}$ and kept for $40 \mathrm{~min}$ to complete phase separation. 
TABLE I. Average photothermal deflection signals for various levels of iron(II) at the spot (average area $2 \mathrm{~mm}^{2}$ ) for the same and different Silufol plates, $532 \mathrm{~nm}, 50 \mathrm{~mW}$. The signal from the untreated sorbent surface was set to zero $(P=0.95)$.

\begin{tabular}{|c|c|c|c|c|c|}
\hline Conditions & Water & Blank & $\begin{array}{c}c_{\mathrm{Fe}}=5 \\
\times 10^{-9} \mathrm{M}\end{array}$ & $\begin{array}{c}c_{\mathrm{Fe}}=1 \\
\times 10^{-7} \mathrm{M}\end{array}$ & $\begin{array}{c}c_{\mathrm{Fe}}=2 \\
\times 10^{-6} \mathrm{M}\end{array}$ \\
\hline Different detection points of the same spot $(n=1000)$ & $0.02 \pm 0.02$ & $3.3 \pm 0.9$ & $25 \pm 5$ & $520 \pm 40$ & $11000 \pm 300$ \\
\hline
\end{tabular}

Next, five portions of $3 \mu \mathrm{L}$ of the aqueous phase were in sequence applied to a Silufol plate using an adjustable plastic pipette (total applied volume of $15 \mu \mathrm{L}$ ). The plate was dried under ambient conditions to dryness prior to photothermal measurements $(532 \mathrm{~nm}, 50 \mathrm{~mW})$ and measured by PDS. If the signal from the spot was negligibly different from the untreated Silufol surface, this ferrozine solution was treated as a stock reagent solution. Otherwise, the extraction procedure was repeated.

Silufol plates (Chemapol, Czech Republic) were used throughout the work. New plates were immersed in Milli-Q water in a teflon vessel for a week, and the water and the sorbent material were tested for the total iron content by atomic-absorption spectroscopy (a Perkin-Elmer 603 atomicabsorption spectrometer, a 100-fold preconcentration); the residual concentration of iron(II) in the sorbent was $(2 \pm 1) \times$ $10^{-13} \mathrm{~mol} / \mathrm{cm}^{2}$ of the plate, which was proved to be at least tenfold lower than the average concentration of iron in the spot of $3 \times 10^{-12} \mathrm{~mol} / \mathrm{cm}^{2}$ achieved as a detection limit for the developed procedure. All the glassware and disposable parts (plastic pipette tips) were treated in the same manner with Milli-Q water in teflon vessels and were considered usable if the signal of a fresh sample of Milli-Q water in a one-hour contact with the plastic or glassware was negligibly different from the initial signal of the water.

All the conditions for iron-ferrozine chelate preparation were selected according to our previous study. ${ }^{29}$

Procedures. Detection limits were estimated according to IUPAC $^{30}$ as a mean square deviation of the blank signal (photothermal-deflection signal from a sample with no iron added) multiplied by a factor of 3 (and multiplied by the calibration plot slope for converting to concentrational units). Quantification limits were estimated as a level of the signal corrected by the blank value when its relative mean square deviation becomes lower than 33\%. Conversion to the concentrational units was made in exactly the same way.

Procedure 1. All the conditions (time of interaction, reagent ratios) were selected according to the previous study. ${ }^{29} \mathrm{~A} 0.5$ $\mathrm{mL}$ portion of the ascorbic acid solution $\left(25 \mathrm{~g} \mathrm{~L}^{-1}\right), 0.5 \mathrm{~mL}$ of the acetic buffer solution, and $0.5 \mathrm{~mL}$ of a ferrozine solution (5 $\times 10^{-4} \mathrm{M}$ ) were added to the necessary amount of iron(III) solution. The range of iron concentrations was $1 \times 10^{-9}$ to $1 \times$ $10^{-5} \mathrm{M}$. Next, the total volume was adjusted with water to 20 $\mathrm{mL}$. Blank sample was prepared in the same way but without adding an iron solution. Five portions of $3 \mu \mathrm{L}$ of the solution were applied in sequence to a Silufol plate using an adjustable pipette (total applied volume of $15 \mu \mathrm{L}$ ). The plate was dried under ambient conditions to dryness prior to photothermal measurements $(532 \mathrm{~nm}, 50 \mathrm{~mW})$.

Procedure 2. A $15 \mu \mathrm{L}$ portion (5 portions of $3 \mu \mathrm{L}$ ) of the mixed solution containing ammonium iron(II) sulfate hexahydrate or iron(III) certified reference material (GSO 7784-2000), with a range of iron concentrations of $1 \times 10^{-9}$ to $1 \times 10^{-5} \mathrm{M}$, and ascorbic acid $\left(25 \mathrm{~g} \mathrm{~L}^{-1}\right)$ was applied to a Silufol plate using an adjustable pipette. Next, a $3 \mu \mathrm{L}$ aliquot of the buffer solution of ferrozine $\left(5 \times 10^{-4} \mathrm{M}\right)$ and ascorbic acid $\left(25 \mathrm{~g} \mathrm{~L}^{-1}\right)$ was applied to the same spot. The plate was dried under ambient conditions to dryness prior to photothermal deflection measurements $(532 \mathrm{~nm}, 50 \mathrm{~mW})$.

\section{RESULTS AND DISCUSSION}

For iron determination, we selected a procedure based on the formation of the iron(II) complex with ferrozine (3-(2-pyridyl)1,2,4-triazine-5,6-bis-(4-phenylsulfonic) acid or its mono- or disodium salts, which have much better solubility in water). This reagent is used for highly sensitive and selective photometric determination of iron, particularly in environmental water samples. ${ }^{29}$ It forms a chelate with iron(II) with the ratio of Fe:ferrozine of 1:3 having an absorption band maximum at $562 \mathrm{~nm}$ and does not react with iron(III). ${ }^{32,33}$ Apparent molar absorptivity of the chelate at the absorption maximum is $\varepsilon_{562}=28000 \mathrm{~L} \mathrm{~mol}^{-1} \mathrm{~cm}^{-1}$, while the absorption of the reagent at this wavelength is negligible. However, the sensitivity of this procedure is insufficient in some cases (i.e., the determination of silt water and seawater ${ }^{34}$ ), and the use of additional preconcentrations becomes necessary. ${ }^{35}$ We selected the excitation laser wavelength of $532 \mathrm{~nm}$ because it is close to the chelate absorption maximum at $562 \mathrm{~nm}$ and a decrease in sensitivity due to lower molar absorptivity is as low as $20 \%$. The chelate was found stable under the action of laser irradiation at the selected excitation wavelength and power for at least $1 \mathrm{~h}$.

Thus, we propose an instrumental approach employing PDS or increasing the sensitivity of the chelate absorption determination. PDS is especially sensitive in the case of surface detection of absorption. Thus, as the first iteration, Procedure 1 includes the following steps: the formation of the colored chelate in solution as in spectrophotometric determination, application of the chelate solution to a Silufol plate, and, finally, photothermal detection of the chelate at the plate using PDS. This made it possible to use the previously found conditions for iron(II) determination ${ }^{29}$ without any changes to focus on the features of PDS.

First, the use of highly sensitive photothermal detection for trace determination of analytes imposes some restrictions. Apart from the requirement of optically homogeneous samples, PDS additionally requires thermal homogeneity of the sample. The studied Silufol plates were found to meet these conditions well. The average signals of the different spots with the same concentration level of iron at the same plate and of the different plates differed insignificantly (Table I).

The sample image of a colored spot of the chelate is presented in Fig. 2a. This image and its lateral profiles (Fig. 2b) were used to further compare photothermal signals. Examining 


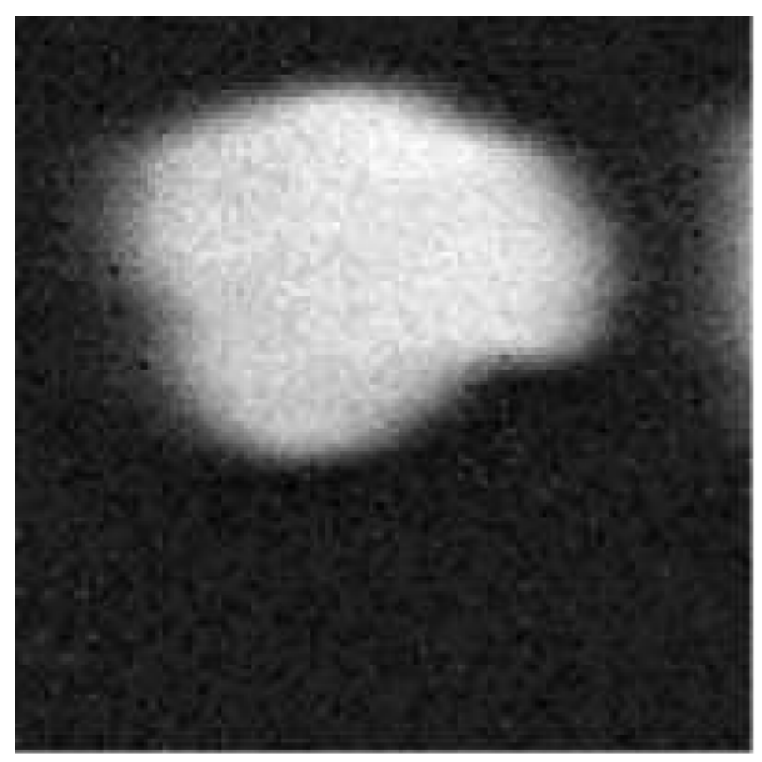

a

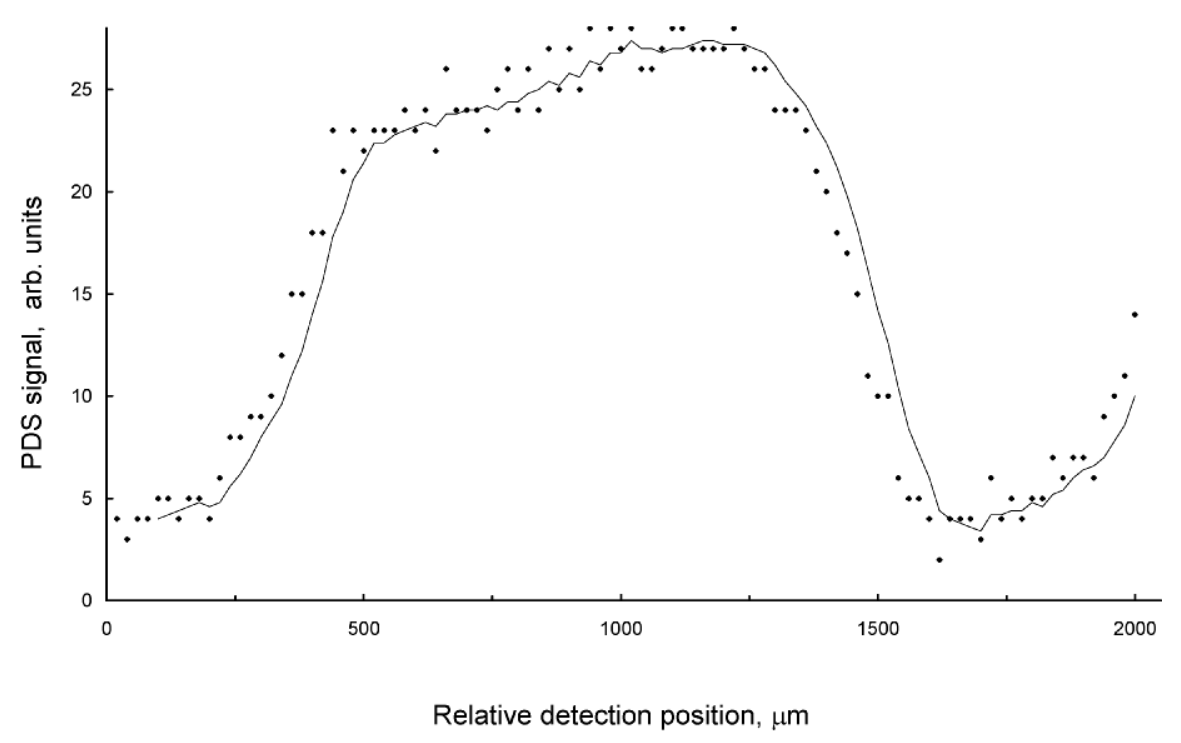

b

FIG. 2. (a) Photothermal image of a spot containing $1.2 \mathrm{pmol}$ of the iron(II) ferrozine chelate. Image area is $2 \times 2 \mathrm{~mm}$, resolution is $100 \times 100$ points, $20 \times 20 \mu \mathrm{m}$ each point; $532 \mathrm{~nm}, P_{\mathrm{e}}=50 \mathrm{~mW}$; (b) photothermal signal profile along the same lateral cross-section of this sample image. Points denote experimental values and the solid line is a moving average of the signal with a period of five points.

signal profiles across the spot for both detection techniques, one can easily distinguish their features for selecting the optimum positioning of the excitation source. We also used an extra signal filter using a moving average with a period of five points. This filtered profile (Fig. 2b) looked smooth and the one-point measurements throughout a spot show good reproducibility (Table I). The slope at the spot edges is sharper, which may result from a nonselective photothermal response of auxiliary reagents concentrated at the spot edge during solution application.

However, Procedure 1 is non-optimal for photothermal detection of real solutions as it can be used for relatively high concentration levels of iron(II) in solution to facilitate its chelation with reagent. However, preconcentration of the initial solution of iron(II) at the substrate followed by the chelation reaction of the preconcentrated iron(II) sample directly at the substrate seems much more promising. However, this means some effort on the optimization of the photometric procedure. Our previous experience in developing photothermal procedures shows the most attention concerning interfering impurities should be paid to the buffer and reducing reagent solutions. ${ }^{36}$ However, in the case of the iron(II) reaction with ferrozine,$^{29}$ the selectivity and high stability constant provide almost no change in the reaction conditions for the reaction in 


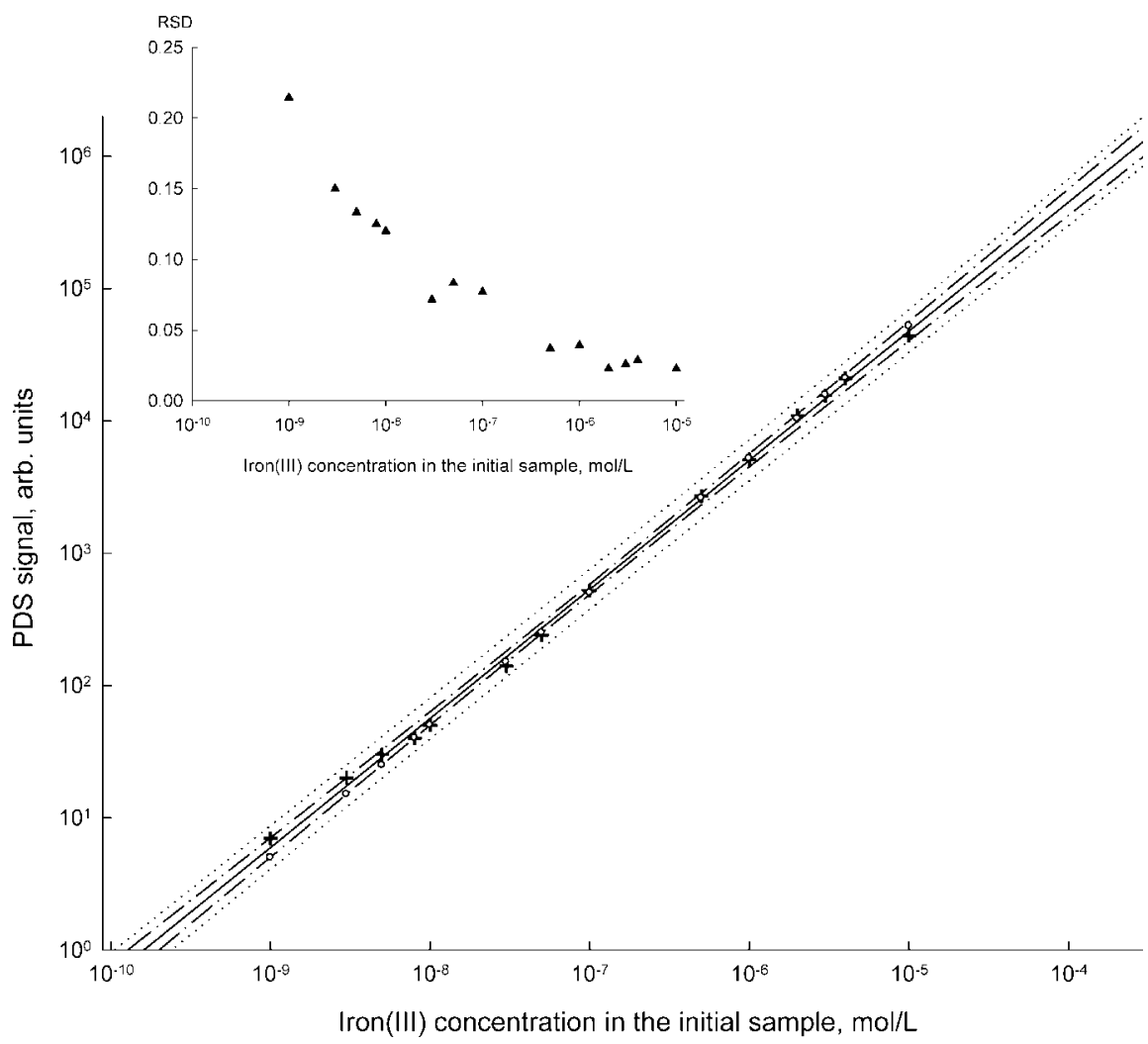

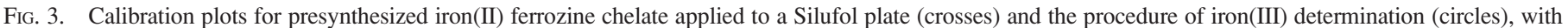

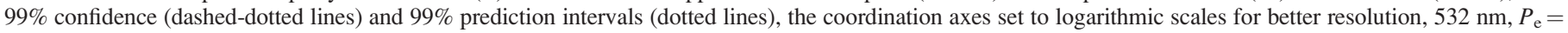
$50 \mathrm{~mW}$. (Inset) The dependence of the RSD of iron(III) determination on the concentration level.

the solid phase compared to the reaction in solution. Thus, Procedure 2 is a very close analogue of Procedure 1 and the spectrophotometric procedure. ${ }^{29}$ For comparison sake, we calculated the performance parameters for both procedures, the reaction in solution followed by sorption preconcentration and the reaction at the substrate. The coefficients of correlation for the linear calibration plots for all the cases exceeded 0.98. The calibration equation for the chelate pre-synthesized in solution is

$$
\begin{aligned}
& \theta=(3.50 \pm 0.02) \times 10^{14} q ; \\
& n=15, P=0.99, r=0.993
\end{aligned}
$$

where $\theta$ (arbitrary units) is the amplitude of the photothermal signal, and $q(\mathrm{~mol})$ is the total chelate amount at the spot. For Procedure 2, the calibration equations are

$$
\begin{aligned}
& \theta=(3.50 \pm 0.02) \times 10^{14} q+(4 \pm 2) ; \\
& n=15, P=0.99, r=0.989
\end{aligned}
$$

for iron(II) and

$$
\begin{aligned}
& \theta=(3.55 \pm 0.03) \times 10^{14} q+(6 \pm 3) ; \\
& n=15, P=0.99, r=0.985
\end{aligned}
$$

for iron(III).

The values of the signals $\theta$ in all three experimental equations are in good agreement with the theoretical amplitude signal calculated from Eq. 1, $\theta=3.62 \pm 0.07$ for $q=10 \mathrm{fmol}$ at the spot (the error of calculation is determined by the precision of measurements of the thermo-optical parameters and laser waist sizes). The differences in characteristics for either procedure used, both with pre-synthesized chelate solutions and with the implementation of colored chelate formation directly at the plate surface, were negligible (Fig. 3), which shows that no significant interfering factors are introduced from the procedures.

The inset in Fig. 3 shows that the RSD of iron(II) determination decreases with an increase in iron(II) concentration, which is described by a rational hyperbolic equation:

$$
\operatorname{RSD}=\frac{(1.6 \pm 0.3)+(3.5 \pm 0.6) \times 10^{-2} c}{(7 \pm 1)+c}
$$

where $c$ is iron concentration in the initial sample, nmol/L. This equation agrees well with the general character of the error curve for PDS ${ }^{23,31}$ with due account of the molar absorptivity of iron and the measurement precision of the instrument used. Thus, no significant interfering factors affect the PDS measurements of iron on plates. Within the linear range of the calibration plot, the RSD value was $0.03-0.20$ and did not exceed 0.10 when the total amount of chelate at the spot was greater than $0.1 \mathrm{nmol}(10 \mathrm{nM}$ in the test solution).

Due to negligible difference in calibration parameters, the performance parameters for both procedures were the same. We achieved an absolute detection limit of $8 \mathrm{fmol}$ in the spot (average concentration of iron in the spot is $3 \times 10^{-12} \mathrm{~mol} /$ $\mathrm{cm}^{2}$ ). In turn, this corresponds to a $0.5 \mathrm{nM}$ chelate concentration in the test solution. The linearity range was 2 
TABLE II. The comparison of application of the same amount of iron(II) (15 pmol) to Silufol plates by $30 \mu \mathrm{L}$ aliquots from the solutions of various concentrations, $532 \mathrm{~nm}, P_{e}=\mathbf{5 0} \mathrm{mW}(P=0.95)$.

\begin{tabular}{ccc}
\hline $\begin{array}{c}\text { Solution } \\
\text { concentration, } \\
\text { mol/L }\end{array}$ & $\begin{array}{c}\text { Number of } \\
\text { successive } \\
\text { applications of } \\
\text { test solutions }\end{array}$ & $\begin{array}{c}\text { Average PDS } \\
\text { signal of the } \\
\text { spot, arb. units }\end{array}$ \\
\hline $2 \times 10^{-8}$ & 1 & $500 \pm 20$ \\
$4 \times 10^{-8}$ & 2 & $510 \pm 30$ \\
$6 \times 10^{-8}$ & 3 & $500 \pm 30$ \\
$1 \times 10^{-7}$ & 5 & $520 \pm 40$ \\
$2 \times 10^{-7}$ & 10 & $540 \pm 50$ \\
$4 \times 10^{-8}$ & 20 & $480 \pm 60$ \\
$1 \times 10^{-6}$ & 50 & $400 \pm 100$ \\
\hline
\end{tabular}

$\times 10^{-18}$ to $3.0 \times 10^{-14} \mathrm{~mol}$ of the chelate at the irradiated zone (single-point measurements) and $1 \times 10^{-14}$ to $2 \times 10^{-10} \mathrm{~mol}$ of the chelate at the spot (imaging experiments), which corresponded to $1 \times 10^{-9}$ to $4 \times 10^{-6} \mathrm{M}$ of the chelate and $3 \times 10^{-5}$ to 0.1 absorbance units in the test solution. The upper linearity limit may be governed by several reasons, including photothermal signal saturation, i.e., the saturation of adsorption leading to a change in the concentration profile at the spot under high chelate concentrations. In this situation, the signal value showed a negative deviation from the linearity without a loss of reproducibility.

The above data were obtained for five successive applications of the solution. However, the studies of Silufol plates showed that the number of successive applications of the test iron(II) solution can be varied in a wide range. Table II shows that the results differ insignificantly when the same quantity of iron is applied as a single $3 \mu \mathrm{L}$ portion of a more concentrated solution or a sequence of twenty applications of a 20-fold less concentrated solution. However, a further increase in the number of applications results in a significant loss of reproducibility of the signal partially due to expansion of the spot and partially due to washout of the sorbent thin layer. Still, even a 50-fold successive application results in rather reproducible though underestimated results. Nevertheless, these experiments showed that the developed approach is flexible: if some prior knowledge of the iron level in the test sample is known, we can either apply the test solution once, thus simplifying the sample preparation, or focus on the preconcentration, thus decreasing the concentration limit of detection.

This procedure uses the same chemical conditions as the previously described spectrophotometric procedure $^{29}$ without

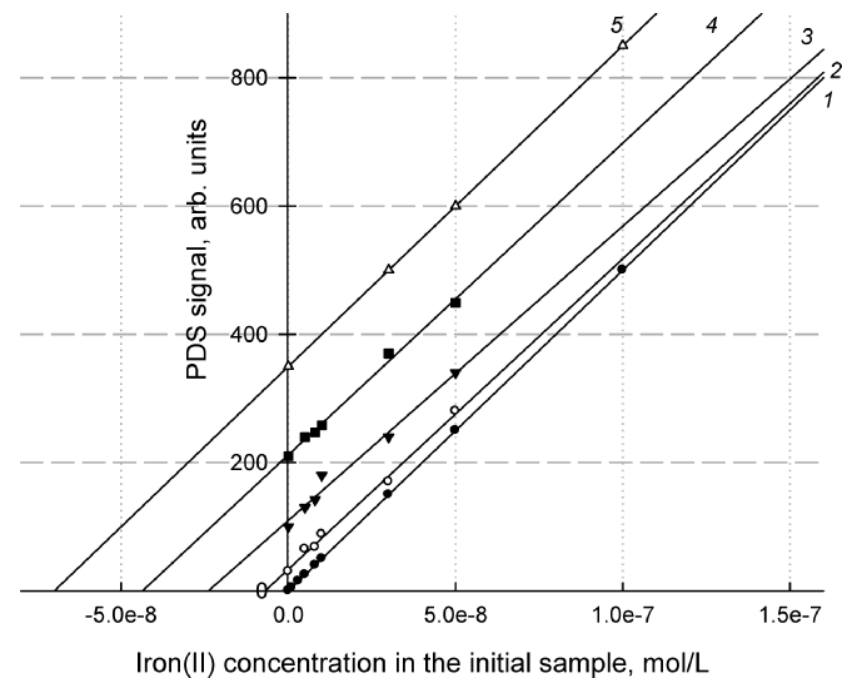

FIG. 4. The photothermal deflection determination of iron(II) with ferrozine by spiked solutions, $532 \mathrm{~nm}, P_{\mathrm{e}}=50 \mathrm{~mW}$. (1) The calibration plot without iron(II), (2) $5 \times 10^{-9} \mathrm{M}$; (3) $2 \times 10^{-8} \mathrm{M}$; (4) $4 \times 10^{-8} \mathrm{M}$; and (5) $5 \times 10^{-9} \mathrm{M}$.

any extra optimization. Hence, the analysis is a simple application of a sample solution to a Silufol plate followed by the application of a reagent solution and does not require any time-consuming or laborious treatment. This is not specific to the PDS detection and can be used with any other analytical methods of surface determination based on molecular spectroscopy, e.g., diffuse reflection spectroscopy. ${ }^{37}$

The procedure also provides a method of sample storage. One can mark sample areas at the plate, apply test solutions and keep samples as long as necessary prior to the analysis, preferably under an inert-gas atmosphere. The experiments with the application of the test iron solution to the plates and performing the procedure immediately and after 1 to 30 days of storage in a refrigerator $\left(-20{ }^{\circ} \mathrm{C}\right)$ shows that the PDS signal of samples with various iron(II) concentrations differs insignificantly for the whole period covered. Under these conditions, an attomolar absolute detection limit at the excitation zone was achieved by PDS, and the linearity range exceeded three orders of magnitude.

The proposed procedure does not require any separate preconcentration stage when treating samples but includes it indirectly as the application of the sample solution to a Silufol plate. In conjunction with a high spatial resolution, this approach provides test solution volumes at the level of $10 \mu \mathrm{L}$, which may actually be reduced even further. This is a very

TABLE III. The determination of iron(II) with ferrozine in neat solutions using spiked solutions by photothermal deflection spectroscopy on Silufol substrate plates, $532 \mathrm{~nm}, P_{e}=50 \mathrm{~mW}(n=3, P=0.95)$.

\begin{tabular}{|c|c|c|c|}
\hline $\begin{array}{l}\text { Iron(II) amount } \\
\text { added, mol }\end{array}$ & $\begin{array}{l}\text { Iron(II) amount } \\
\text { found, mol }\end{array}$ & Concentration, $\mathrm{mol} / \mathrm{L}$ & $\begin{array}{c}\text { Concentration, } \\
\text { determined by AAS }\end{array}$ \\
\hline $5 \times 10^{-14}$ & $(6 \pm 1) \times 10^{-14}$ & $(3.0 \pm 0.5) \times 10^{-9}$ & $(3 \pm 1) \times 10^{-9}$ \\
\hline $3 \times 10^{-13}$ & $(4 \pm 1) \times 10^{-13}$ & $(3.0 \pm 0.7) \times 10^{-8}$ & $(3.1 \pm 0.5) \times 10^{-8}$ \\
\hline $1 \times 10^{-12}$ & $(1.1 \pm 0.2) \times 10^{-12}$ & $(9 \pm 1) \times 10^{-8}$ & $(10 \pm 2) \times 10^{-8}$ \\
\hline $5 \times 10^{-12}$ & $(4.8 \pm 0.2) \times 10^{-12}$ & $(4.0 \pm 0.2) \times 10^{-7}$ & $(3.6 \pm 0.3) \times 10^{-7}$ \\
\hline
\end{tabular}


TABLE IV. The determination of iron(II) with ferrozine in artificial solutions using spiked solutions by photothermal deflection spectroscopy on Silufol substrate plates, $532 \mathrm{~nm}, P_{e}=50 \mathrm{~mW}(n=3, P=0.95)$.

\begin{tabular}{lrrr}
\hline \multicolumn{1}{c}{ Conditions } & $\begin{array}{c}\text { Iron(II) concentration } \\
\text { added, mol/L }\end{array}$ & $\begin{array}{c}\text { Iron(II) concentration, } \\
\text { found, mol/L }\end{array}$ & $\begin{array}{c}\text { Concentration, } \\
\text { determined by AAS }\end{array}$ \\
\hline Doubly distilled water with $1 \mathrm{M} \mathrm{NaCl}$ & $3 \times 10^{-8}$ & $(3.0 \pm 0.6) \times 10^{-8}$ & $(3.0 \pm 0.5) \times 10^{-8}$ \\
& $8 \times 10^{-8}$ & $(8.5 \pm 0.5) \times 10^{-8}$ & $(8 \pm 1) \times 10^{-8}$ \\
& $3.5 \times 10^{-7}$ & $(3.6 \pm 0.3) \times 10^{-7}$ & $(3.6 \pm 0.3) \times 10^{-7}$ \\
Doubly distilled water with $1 \times 10^{-6} \mathrm{M} \mathrm{Cu}^{2+}$ & $3 \times 10^{-8}$ & $(8.2 \pm 0.5) \times 10^{-8}$ & $(2.9 \pm 0.4) \times 10^{-8}$ \\
& $3.5 \times 10^{-8}$ & $(3.8 \pm 0.4) \times 10^{-8}$ & $(8 \pm 1) \times 10^{-8}$ \\
Doubly distilled water with $1 \times 10^{-6} \mathrm{M} \mathrm{Cu}^{2+}$ and $1 \mathrm{M} \mathrm{NaCl}$ & $3 \times 10^{-8}$ & $(3.1 \pm 0.6) \times 10^{-8}$ & $(3.7 \pm 0.3) \times 10^{-7}$ \\
& $8 \times 10^{-8}$ & $(8.0 \pm 0.4) \times 10^{-8}$ & $(3.1 \pm 0.5) \times 10^{-8}$ \\
& $3.5 \times 10^{-7}$ & $(3.6 \pm 0.5) \times 10^{-7}$ & $(3.5 \pm 0.3) \times 10^{-8}$ \\
\hline
\end{tabular}

important feature when available amounts of test samples are very limited.

Procedure 2 was verified using spiked solutions (Fig. 4) and showed good linearity and no significant deviation from the slope of an unspiked curve. The data obtained (Table III) show no bias of the results obtained for a wide range of iron(II) concentrations.

The aim of this paper was to test the applicability of the approach of a combination of a spectrophotometric procedure at a substrate with photothermal deflection. Thus, a thorough testing of the procedure was out of the scope of this work. Still, we checked the performance parameters of PDS determination of iron for various interfering conditions (high saline background or (and) significant amounts of copper as an interfering element (Table IV)) and found no significant errors in the experiment.

The comparison with the existing procedures ${ }^{17,18}$ shows that the developed procedure shows a significant increase in the sensitivity: while the absolute amount of Fe that could be determined by Refs. 17 and 18 is $1 \mathrm{pmol}(0.05 \mathrm{nM}$ in $20 \mathrm{~mL})$, a decrease in the absolute limit of detection is more than 100fold: $1 \mathrm{pmol} / 8 \mathrm{fmol}=125$. Note that we compare the 'balanced' procedure with five successive applications of the test solution: as we mentioned above, we may increase the number of applications of the test solution without a loss of reproducibility. Hence, the absolute limit of detection will be the same, but the concentration LOD will be at the same level as in Refs. 17 and 18, but only for $3 \mu \mathrm{L} \times 50=0.15 \mathrm{~mL}$ of the total sample.

However, in fact, the flow-injection procedure ${ }^{17,18}$ has the important advantage of real-time monitoring and can be used for rapid on-site analysis of iron. To the contrary, the developed procedure, with the feature of long-term stability of stored samples, can be used for a more detailed in-laboratory analysis of the samples with the increased sensitivity of the selected samples. Thus, the procedures complement each other.

The obtained analytical characteristics appear very advantageous for trace determination of iron. These performance characteristics are perfectly supplemented with a very low sample volume of $15 \mu \mathrm{L}$, which is very important for trace analysis under low available sample amounts. However, thoroughly studying sample matrix effects and carefully changing chemical conditions (buffer concentration and composition, selection of reducing and masking reagents, etc.) compared to the original spectrophotometric procedure may result in additional improvement ${ }^{14}$ of sensitivity and selectivity of the PDS analysis.

\section{CONCLUSION}

We have thus employed PDS for the detection of a colored analyte on a sorbent thin-layer plate under the optical excitation selective to the absorbed substance with good performance parameters. We can recommend this photothermal technique for highly sensitive molecular-absorption determination of colored analytes adsorbed on sorbents, in particular, for sensitive detection in thin-layer chromatography and similar solid-phase analyses of absorbing substances. The additional possibility of sample storage specified by the proposed procedure may also be useful when test samples cannot be analyzed in situ and must be carried to a laboratory.

\section{ACKNOWLEDGMENTS}

The authors would like to thank Stefan Heissler for very helpful technical assistance and valuable discussions in performing the photothermal beam deflection experiments. This study was partially supported by the Russian Foundation for Basic Research, project no. 07-03-00859-a.

1. T. Ikari, J. P. Roger, and D. Fournier, Rev. Sci. Instrum. 74, 553 (2003). 2. A. C. Boccara, D. Fournier, and J. Badoz, Appl. Phys. Lett. 36, 130 (1980).

3. S. Janssen, G. R. Hofmann, W. Faubel, and H. J. Ache, Fresenius' J. Anal. Chem. 360, 788 (1998).

4. M. Bertolotti, G. L. Liakhou, R. Li Voti, S. Paoloni, and C. Sibilia, Appl. Phys. B 67, 641 (1998).

5. M. J. S. Kreiter and S. Mittler-Neher, Thin Solid Films 342, 244 (1999).

6. K. Plamann, D. Fournier, B. C. Forget, and A. C. Boccara, Diamond Relat. Mater. 5, 699 (1996).

7. M. Harada, S. Obata, T. Kitamori, and T. Sawada, Anal. Chem. 65, 2181 (1993).

8. C.-Y. Tu, T. Kitamori, T. Sawada, H. Kimura, and S. Matsuzawa, Anal. Chem. 65, 3631 (1993).

9. H. Kimura, S. Matsuzawa, C.-Y. Tu, T. Kitamori, and T. Sawada, Anal. Chem. 68, 3063 (1996).

10. F. K. Fotiou and M. D. Morris, Anal. Chem. 59, 185 (1987).

11. F. K. Fotiou and M. D. Morris, Anal. Chem. 59, 1146 (1987).

12. P. J. Treado and M. D. Morris, Appl. Spectrosc. 42, 1487 (1988).

13. J. Gibkes, I. Vovk, J. Bolte, D. Bicanic, B. Bein, and M. Franko, J. Chromatogr., A 786, 163 (1997).

14. M. A. Proskurnin and V. V. Chernysh, J. Anal. Chem. 56, 943 (2000).

15. M. Gledhill, C. M. G. van den Berg, R. F. Nolting, and K. R. Timmermans, Mar. Chem. 59, 283 (1998).

16. C. M. G. van den Berg, M. Nimmo, O. Abollino, and E. Menasti, Electroanalysis 3, 477 (1991).

17. H. Obata, H. Karatani, and E. Nakayama, Anal. Chem. 65, 1524 (1993).

18. H. Obata, H. Karatani, M. Matsui, and E. Nakayama, Mar. Chem. 56, 97 (1997).

19. S. Bonnet and C. Gueiu, Geophys. Res. Lett. 31, L03303 (2004).

20. B. D. Hanh, W. Faubel, S. Heissler, S. Wartewig, and R. H. Neubert, Laser Phys. 16, 794 (2006).

21. A. Salnick, W. Faubel, H. Klewe-Nebenius, A. Vendl, and H. J. Ache, Corros. Sci. 37, 741 (1995).

22. A. Salnick and W. Faubel, Appl. Spectrosc. 49, 1516 (1995). 
23. S. E. Bialkowski, Photothermal Spectroscopy Methods for Chemical Analysis (Wiley, New York, 1996).

24. V. P. Zharov and V. S. Letokhov, Laser Optoacoustical Spectroscopy (Springer-Verlag, Berlin, 1986)

25. CRC Handbook of Chemistry and Physics (CRC Press, Boca Raton, FL, 1998), 79th ed.

26. L. A. Oshin, Ed., Promyshlennye Khloroorganicheskie Produkty. Spravochnik [Industrial Organochlorine Products. A Handbook] (Khimiya, Moscow, Russia, 1978).

27. N. V. Chekalin, N. B. Zorov, A. S. Kochetkova, and S. Y. Karpova, Vysokochistye Veshchestva [High Purity Substances] (Khimiya, Moscow, Russia, 1992)

28. V. A. Filichkina, A. G. Abroskin, M. A. Proskurnin, and V. M. Savostina, J. Anal. Chem. (Russ.) 47, 1038 (1992).

29. M. Y. Kononets, S. V. Pakhomova, A. G. Rozanov, and M. A. Proskurnin, J. Anal. Chem. (Russ.) 57, 586 (2002).
30. J. Inczedy, T. Lengyel, and A. M. Ure, Eds., Compendium of Analytical Nomenclature, The Orange Book (Blackwell Science, New York, 1998), 3rd ed.

31. K. Doerffel, Statistics in Analytical Chemistry [Russian translation from German] (Mir, Moscow, Russia, 1969)

32. L. L. Stookey, Anal. Chem. 42, 779 (1970).

33. C. R. Gibbs, Anal. Chem. 48, 1197 (1976).

34. D. W. King, J. Lin, and D. R. Kester, Anal. Chim. Acta 247, 125 (1991).

35. S. Blain and P. Treguer, Anal. Chim. Acta 308, 425 (1995).

36. V. V. Chernysh, M. Y. Kononets, M. A. Proskurnin, S. V. Pakhomova, V. V. Komissarov, and A. I. Zatsman, Fresenius' J. Anal. Chem. 369, 535 (2001).

37. S. B. Savvin, V. P. Dedkova, and O. P. Shvoeva, Russ. Chem. Rev. 69, 187 (2000). 